\title{
Comparing EEG analyses with different epoch alignments in an auditory lexical decision experiment
}

\author{
Louis ten Bosch ${ }^{1}$, Kimberley Mulder $^{1}$, Lou Boves $^{1}$ \\ ${ }^{1}$ Center for Language Studies, Radboud University, Nijmegen, The Netherlands \\ l.tenbosch@let.ru.nl,K.Mulderedonders.ru.nl, L.Boves@ru.nl
}

\begin{abstract}
In processing behavioral data from auditory lexical decision, reaction times (RT) can be defined relative to stimulus onset or relative to stimulus offset. Using stimulus onset as the reference invokes models that assumes that relevant processing starts immediately, while stimulus offset invokes models that assume that relevant processing can only start when the acoustic input is complete. It is suggested that EEG recordings can be used to tear apart putative processes. EEG analysis requires some kind of time-locking of epochs, so that averaging of multiple signals does not mix up effects of different processes. However, in many lexical decision experiments the duration of the speech stimuli varies substantially. Consequently, processes tied to stimulus offset are not appropriately aligned and might get lost in the averaging process. In this paper we investigate whether the time course of putative processes such as phonetic encoding, lexical access and decision making can be derived from ERPs and from instantaneous power representations in several frequency bands when epochs are time-locked at stimulus onset or stimulus offset. In addition, we investigate whether time-locking at the moment when the response is given can shed light on the decision process per sé.

Index Terms: EEG analysis, ERP, instantaneous power, epoch time-lock, linear mixed effects models, lexical decision, cognates
\end{abstract}

\section{Introduction}

In auditory lexical decision experiments reaction times (RTs) can be defined in two ways: as the time between stimulus onset and some overt reaction, or the time between stimulus offset and the reaction. Rarely, if ever, the decision about the RT's starting point is motivated with references to putative cognitive processes that operate during the processing of a stimulus. When only behavioural data are available, such as correct/wrong decisions and RTs, there is no way in which putative underlying processes can be separated: the time it takes to make a decision is the accumulated result of all perceptual, cognitive and executive processes. Processes that are implicated in auditory lexical decision include at least form recognition, lexical access, decision making and action. Older models assumed that the processes were self-contained modules and that they operated sequentially, e.g., [1, 2]. However, there is increasing evidence that several processes may operate simultaneously, with complex mutual interactions e.g., $[3,4,5]$.

If RT is defined relative to stimulus onset, it is (tacitly) assumed that form recognition starts immediately after stimulus onset, even if it will probably take some time before enough information is available to select a number of promising cohorts. If RT is defined relative to stimulus offset a (tacit) assumption is that form recognition is complete at offset.

There is an increasing number of papers that use EEG or
MEG recordings in attempts to disentangle the time courses of the cognitive processes in auditory lexical decision tasks (e.g. $[6,7,8])$. In most studies, E/MEG epochs are time-locked to the moment of stimulus onset, and baseline correction is applied on a short time interval preceding that moment. However, it can be argued that there are other events to which epochs can or should be time-locked. For example, [9] investigated timelocking to the moment of the uniqueness point in words and the deviation point in pseudo-words. While it seems easy to define uniqueness and deviation points on the basis of phonemic transcriptions of stimulus words, we adhere to a computational model of spoken word recognition that does not involve discrete segmental pre-lexical representations [10]. For that reason, we believe that it is not possible to accurately define these moments in actual acoustic stimuli. We prefer to use an unambiguous event, namely the offset of the acoustic stimuli as an alternative time-lock moment. Time-locking on stimulus offset may be especially revelatory in experiments that use acoustic stimuli with a wide range of durations.

Almost invariably, reaction times in auditory lexical decision experiments are much longer than the physical duration of the stimuli. This raises the question whether time-locking to stimulus onset or offset is adequate for investigating the decision process that operates between the moment when no additional acoustic information becomes available and the actual decision moment. In research that is focused on the decision process it is proposed to time-lock brain signals to the decision moment (e.g., [11, 12].

In this paper we use data from a lexical decision experiment [13] in which highly proficient Dutch learners of English classified speech stimuli as English words or pseudo-words (e.g., faliche, bammer. Their task was complicated by two manipulations: First, part of the word stimuli had a weak syllable that was heavily reduced. Moreover, half of the reduced stimuli had a weak-strong-weak (WSW) stress pattern with the first syllable reduced (e.g., /p'fesər/ for professor), while the other half had a strong-weak-weak (SWW) pattern with the middle syllable reduced (e.g. I'ssmri/ for summary). Finally, half of the target stimuli were Dutch-English cognates (professor, for example, is a word that has the same meaning in Dutch and English).

Previous analyses of the data (e.g., [13]) were focused on the effects of the cognate status of the stimuli, and the effect of reduction. In this paper, we treat all stimulus types on an equal basis. Therefore, we will consider full and reduced cognates and full or reduced controls as independent types. As a result, we will analyze the differences between six stimulus types in both the SWW and WSW conditions.

In addition to conventional ERPs studies, the literature contains a growing number and variety of investigations of oscillations in a number of frequency bands. Still, it is fair to say that the interpretations of the observations can only be speculative, mainly due to the absence of rigid physical models of 
the processes that generate the EEG signals. Often the interpretations depend on psycholinguistic models that are themselves under discussion. For example, the interpretation of oscillations in the theta band in [14] are based on theories of spoken word comprehension that assume a discrete abstract pre-lexical representation (e.g. [15]), an assumption that is challenged by $[10,16]$. [17] discuss a large number of seemingly contradictory experimental results against the background of two competing (oscillatory- or gain-based) models of neural activity.

\section{Method}

Forty right-handed Dutch master students of English-taught courses at Radboud University (mean age: 20.9 years) participated in the experiment. EEG recordings of 29 were deemed suitable for in-depth analysis. Participants heard 900 experimental stimuli in a session that was split in two sub-sessions, one dedicated to WSW stimuli, the other to SWW stimuli. Half of the stimuli were pseudo-words. The words consisted of 68 cognates and 68 matched controls for the WSW block and 48 cognates and matched controls for the SWW block. The remaining stimuli were filler words. The cognates and controls had similar log subtitle word frequencies in British-English corpora ([18]; mean frequency in the WSW condition: 2.25 and 2.08; in the SWW condition: 2.18 and 2.41.

EEG signals were recorded using a 64 channel actiCAP, with a sampling frequency of $1 \mathrm{kHz}$. The continuous EEG was segmented into time-locked epochs, in three ways: (a) starting $200 \mathrm{~ms}$ before target word onset, (b) starting $200 \mathrm{~ms}$ before stimulus offset and (c) starting $2000 \mathrm{~ms}$ before the response, which consisted of pressing one of two buttons. The $200 \mathrm{~ms}$ before the event were used for baseline correction. Artifacts were rejected automatically with a procedure that implemented the rejection criteria in the BrainVision ${ }^{\odot}[19]$ software with default values for all four criteria.

In this paper we are only interested in endogenous processes. For that reason we removed the exogenous component of the EEG signals by subtracting the $m T R F$ estimate [20,21] for the onset and offset alignments.

The literature on features of EEG signals in individual frequency bands focuses on the power in a specific (narrow) band (e.g. [8]) or phase coherence in narrow bands (e.g. [22, 23]). To facilitate the interpretation of phenomena in separate frequency bands, we attempted to create signal representations similar to ERPs in the full frequency band. In this paper we focus on the instantaneous power of the spectral components of EEG signals in the delta $(1 \leq f \leq 4 \mathrm{~Hz})$, theta $(4 \leq f \leq 8 \mathrm{~Hz})$, alpha $(8 \leq f \leq 12 \mathrm{~Hz}$ and beta $(12 \leq f \leq 20 \mathrm{~Hz})$ frequency bands. To isolate the frequency bands we used $6^{\text {th }}$ order zero phase Butterworth filters, while the instantaneous power was defined as the square of the absolute value of the Hilbert transform. For the delta band (but not for the other frequency bands) it is possible to obtain useful ERP representations; therefore, we investigated both ERP and instantaneous power in the delta band. Before averaging the instantaneous power traces, we applied a baseline correction similar to what is usual in computing ERPs: we subtracted the average power in the time interval $200 \mathrm{~ms}$ preceding stimulus onset.

\subsection{Procedure}

For the time-lock on stimulus onset and offset we focus on unraveling effects of the cognate status of a stimulus, the effects of reduction and differences between words and pseudo-words.
For the alignment on the decision moment we focus on differences between words and pseudo-words, and a possible effect of how difficult stimuli are for a lexical decision task. All statistical analyses were performed on EEG signals downsampled to $100 \mathrm{~Hz}$

We started the analyses by generating scalp topography plots that summarize the differences between the six stimulus types in the SWW and in the WSW conditions. For this purpose, we used the conover procedure in the Python package scikit-posthocs to compute sample-based post-hoc contrasts between the six stimulus types for all EEG channels that contained a sufficient number of artefact-free signals for all stimulus types. This enabled us to identify the sensors that were most likely to discriminate between the stimulus types at different points in time. An example is shown in Figure 1. Subsequently, we subjected the channels and time intervals with the largest post-hoc contrasts to a detailed statistical analyses, using a shifting window approach $[24,25]$ with linear mixed effects models [26] in $R$ version 3.5.1 [27]. For obvious reasons it is not possible to search for the optimal lmer model for each individual channel and time interval. Therefore, all analyses were performed with the same model:

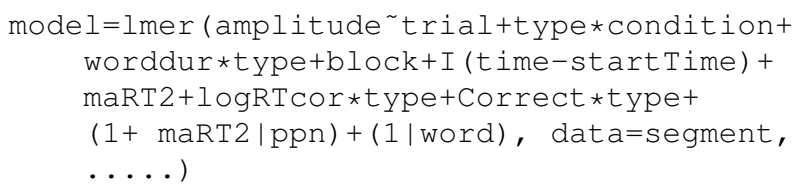

In this model the factor type has six levels (control-full, control-reduced, cognate-full, cognate-reduced, filler, pseudoword), the factor condition has two levels (SWW and WSW). The factor maRT2 summarizes the effects of preceding stimuli and logRTcor is a logarithmic representation of filtered reaction times [28]. We included the factor Correct because it appeared to improve the model fit substantially, in line with the finding that $\log (\mathrm{RT})$ distributions of correct and wrong responses are different [29].

\section{Results}

In this paper we will limit ourselves to the presentation of the results that are most interesting in the context of L2 lexical decision experiments. One general finding, which may apply to all experiments with EEG, is that there are hardly any differences between the stimulus types later than approximately $600 \mathrm{~ms}$ after the time-lock point. This is probably due to the fact that whatever processes may be synchronized at time-lock, they lose their synchrony after more than half a second. The other general finding is that we always see a large difference between the SWW and the WSW condition. We hesitate to link that finding to different representations in the mental lexicon as proposed by [30]. Probably, the presence of a substantial number of stimuli with a heavily reduced leading syllable has made the SWS condition more difficult, reflected in much larger proportions of errors in the lexicality judgments, which was also found by [31]. A final general finding which is clear from Figure 1 is that the location on the scalp where the stimulus types differ most changes over time.

\subsection{Time-lock at stimulus onset}

\subsubsection{ERP}

In general, the ERP patterns for the SWW and WSW conditions are similar, but invariably there is a time shift between 

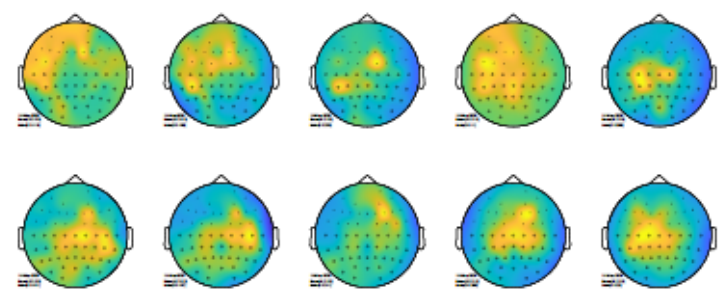

(f) $300 \mathrm{~ms}$

(g) $400 \mathrm{~ms}$

(h) $500 \mathrm{~ms}$

(i) $600 \mathrm{~ms}$

(j) $700 \mathrm{~ms}$
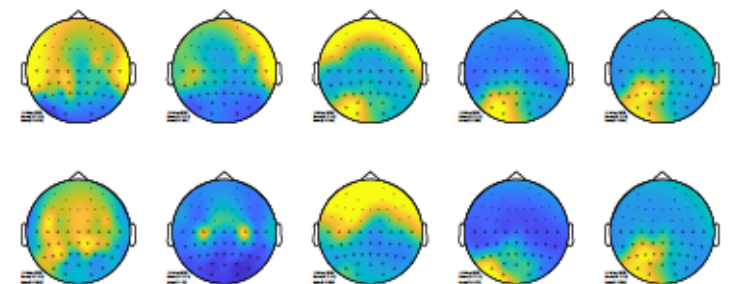

(p) $300 \mathrm{~ms}$

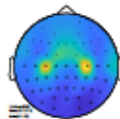

(q) $400 \mathrm{~ms}$
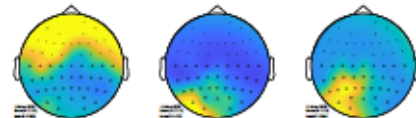

(s) $600 \mathrm{~ms}$

Figure 1: Scalp topographies for ERPs in the delta band. Top panel: time-lock at stimulus onset; bottom panel: time-lock at stimulus offset. In both panels the upper row corresponds to the $S W W$ condition, and the lower row to the WSW condition. Times are 300, 400, 500, 600, $700 \mathrm{~ms}$ after the time-lock event.

the two conditions: the WSW patterns lag the SWW patterns by $100 \mathrm{~ms}$. This is in line with the fact that salient acoustic information in the SWS stimuli comes only in the second syllable. Consequently, cognitive processes that depend on reliable acoustic input can only start later. Another general finding is that the most conspicuous contrasts are not between words and pseudo-words, but between the reduced stimuli and the rest. The pseudo-words only stand out in the traces for P1, P 5, $\mathrm{P} 7, \mathrm{CP} z, \mathrm{CP} 3$, always with a smaller negative amplitude in the time interval centred at $600 \mathrm{~ms}$ after stimulus onset. The distance between pseudo-words and words is larger in the SWW than in the WSW condition.

\subsubsection{Instantaneous power}

The most striking observation from the instantaneous power traces in the delta band is substantial power suppression in the reduced stimuli in the WSW condition. While the immediate effect of the exogenous activity is removed by the mTRF correction in the ERP traces, the effect of reductions in the first syllable remains in the instantaneous power traces, centered around $200 \mathrm{~ms}$ after stimulus onset. The reduced WSW stimuli seem to tax form processing more than the phonotactically legal pseudowords.

In the theta band the global power suppression in the time interval around $200 \mathrm{~ms}$ after onset is clearly diminished. The most conspicuous observation in the theta band is a power enhancement around $400 \mathrm{~ms}$ after onset for the reduced stimuli, especially in the SWW condition. This tracks the location of the reduced syllable in the speech input. The effect is most apparent in the central and frontal areas. Again, this suggests that reduction affects form processing.

The instantaneous power traces in the alpha band only show a tendency towards power enhancement for the reduced stimuli, especially in the SWW condition. The enhancement comes some $50 \mathrm{~ms}$ later than in the theta band, and is it more concen-

trated in the central area.

The traces in the beta band are more difficult to interpret, because they appear to be quite fickle. One interesting observation is the return of a clear power suppression in the reduced WSW stimuli, at around $200 \mathrm{~ms}$ after onset.

What is probably the most striking observation from the analysis of instantaneous power with time-lock at stimulus onset is that the reduced stimuli are the ones that clearly stand out, while the pseudo-words overlap almost completely with the full stimuli (including the fillers). Here, effects of the cognate status are minor or absent.

\subsection{Time-lock at stimulus offset}

Time-locking at stimulus offset should mitigate the effects of the substantial difference in the duration of the stimuli in the analyses of processes involved in accessing semantic information and decision making.

\subsubsection{ERP}

A quite unexpected result from the analysis of ERP traces in the delta band is the difference between the full controls and cognate stimuli and all other types in the time interval up to $400 \mathrm{~ms}$ after stimulus offset in the SWW condition. The target stimuli reach their maximum negative amplitude almost $200 \mathrm{~ms}$ earlier than the other types in all brain areas, except the occipital. A probable explanation is the prosodic similarity between the stimuli in these types. A second general finding is that in the WSW condition the traces for the reduced cognates systematically lag those of the reduced controls by some $50 \mathrm{~ms}$, with a larger negative amplitude around $350 \mathrm{~ms}$ post offset. The traces for the pseudo-words only stand out in $\mathrm{P} 1, \mathrm{P} 3, \mathrm{CP} 1, \mathrm{O} 1$ between 250 and $400 \mathrm{~ms}$ post offset with a flatter pattern in the SWW condition. In the WSW condition the special status of the pseudo-words is clearest in the cluster C1, C3, C5 around $600 \mathrm{~ms}$ post offset. These findings suggest a left-hemisphere effect in a time interval that might be related to semantic access.

\subsubsection{Instantaneous power}

In the SWW condition there were hardly any conspicuous patterns in the instantaneous power traces. In CP 4 there is power enhancement for the pseudo-words and power suppression for the target words around $400 \mathrm{~ms}$ post offset. C5 shows power enhancement for pseudo-words in a long interval from 200 to $600 \mathrm{~ms}$, without clear differences between other types. The effects are much larger in the WSW condition. All channels around $\mathrm{POz}$ show power enhancement for reduced cognates, reduced controls and full cognates, in that order, from $200 \mathrm{~ms}$ post offset onward. This suggests that besides reduction -for somewhat obvious reasons- also cognate status affects what is presumably semantic access.

In the theta band there are no conspicuous differences between the conditions. The only general observation is a tendency for the reduced stimuli (both WSW and SWS, but the latter a bit more) to have higher power in the time interval after $200 \mathrm{~ms}$ post offset.

In the alpha band we see a large power enhancement for the pseud-words in P1, P3, P 5, P 7 in the time interval between 200 and $600 \mathrm{~ms}$ post offset in the SWW condition. In the WSW condition there is a tendency for the reduced stimuli to have higher power, in P 5 joined with the pseudo-words in the same time interval. This might be interpreted as a sign that semantic access causes problems with the pseudo-words. For the 
reduced stimuli semantic access is only especially difficult in the WSW condition.

In the beta band there are no clear patterns, except for some tendency for the reduced stimuli to have higher power between 200 and $500 \mathrm{~ms}$ post offset in the WSW condition.

In summary, with time-lock at stimulus offset we see an unexpected effect of stimuli that shape a specific prosodic pattern. We also see relatively late issues with semantic access for the pseudo-words and the reduced WSW stimuli.

\subsection{Time-lock at button press}

The response in the form of a button press is undoubtedly an 'event'. Several researchers have investigated EEG signals leading up to that event. For that purpose, baseline correction based on a short time interval following the button press is considered appropriate. Using tasks that require participants to accrue information for making a decision, [11, 12] found that straightforward ERPs, based on the average of the sensors $C P 1, C P z$ and $C P 2$ (the centro-parietal positivity or CPP) allow discriminating between correct and incorrect judgments, and fast or slow responses. In research into the impact of feedback on the correctness of decisions, [32] found a clear error- and feedbackrelated negativity in electrode $F z$.

To the best of our knowledge, all research related to the moment when the decision is externalized is based on EEG signal amplitude. Therefore, we will not investigate instantaneous power in this case. Also, we will only investigate whether we can reproduce the results from [11] and [12] in a task in which participants got no feedback about their performance.

Figure 2 shows the average CCP traces as in [11], plus the standard error of the estimates for two subsets of the data. Baseline correction was applied in the interval $-100<$ time $<$ $0 \mathrm{~ms}$ preceding the moment of button press. The top panel contains the data for the part of the experiment with SWW items; the bottom panel contains the corresponding traces for the WSW items. Each panel contains six traces, for full controls and cognates, reduced controls and cognates, fillers and pseudo words (the yellow traces). The traces for the pseudowords stand apart from the other types in the complete time interval from $600 \mathrm{~ms}$ before the button press onward.

The individual traces that together form the ERP traces in Fig. 2 can be characterized by their slope in the time interval between $400 \mathrm{~ms}$ preceding the reaction to the moment the decision is expressed. We defined that slope as the angle (in radians) of the line that connects the largest extremum in that time interval with the value at the moment of button press. An lme 4 model with the predictors task, stimulus type, Log(RT), maRT2 and Correct failed to discriminate between the stimulus types. This suggests that the simple slope measure does not capture important characteristics of the traces.

An lme 4 analysis of the traces in the time interval [-400, -100] ms before the moment of button press with the same predictors yields highly significant results for the factors task, type, maRT2, $\log (\mathrm{RT})$, correct. With full form cognates on the intercept and the interaction type * Correct, reduced cognates and pseudo words differ significantly, but the $t$-values for full and reduced controls and fillers are not significant. This suggests that the decision processes involved with reduced cognates and pseudo-words are different from the corresponding processes for the other stimulus types. The very significant $t$-values for $\log (R T)$ and $m a R T 2$-both with positive $\beta$ - and Correct -with a negative $\beta$ - show that longer RTs and carry-over from preceding stimuli flatten the slope of the CPP.
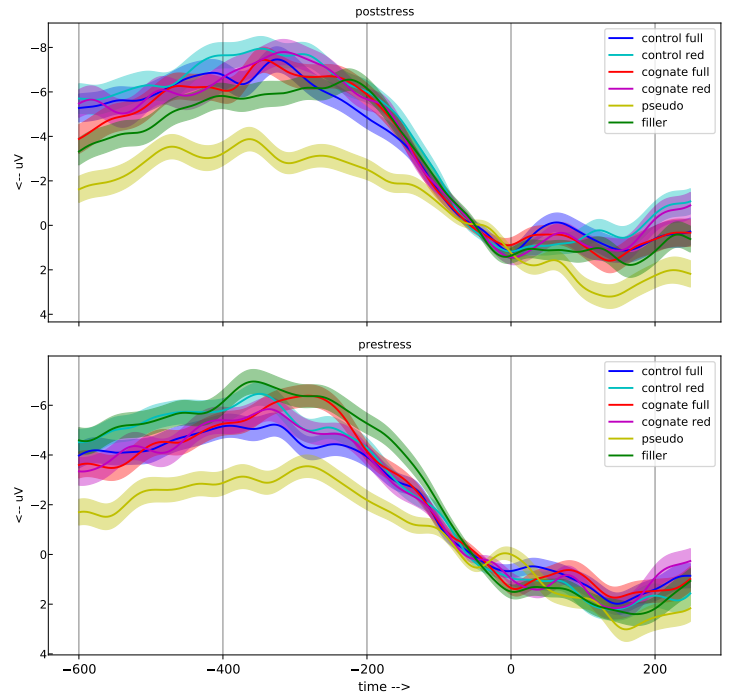

Figure 2: CPP traces for full band signals, baseline correction in the interval 100 ms before decision moment. Top: SWW items, bottom: WSW items.

Correct decisions steepen the slope of the PCC, even if there is no feedback. Apparently, the majority of the correct decisions is easier to make than most wrong decisions. However, the slope of the PCC of an individual stimulus is insufficiently powerful to predict the correctness of the decision for that stimulus.

Also, we were unable to predict the correctness of individual decisions on the basis of the features that [32] derived from the sensor $F z$. It is not clear whether that failure is due to larger variability in our data, or to the fact that the effect observed in [32] only manifests in a design in which participants get feedback on their performance.

\section{Conclusions}

In this paper we used the EEG data from a lexical decision experiment [13] that aimed to investigate the impact of speech reduction and cognate status. By time-locking EEG epochs at stimulus onset and offset, and at the moment the decision was expressed, we were able to show that before the end of the acoustic stimuli there is no effect of pseudo-words; only reduced stimuli stand out, especially in instantaneous power in the beta band. Time-locking at stimulus offset shows that reduced forms have similar issues with semantic access as pseudowords. Only time-locking at the moment of button press showed a clear difference between words and pseudo-words. Taken together, these results suggest that the decision process depends heavily on how easy it is to find a meaning, and that eventually pseudo-words lose out against reduced forms.

Intriguingly, we found a clear effect of cognate status in the WSW condition in the ERP signals time-locked at stimulus offset. This may be related to an interference caused by a simultaneous -but late- activation of $\mathrm{L} 1$ and $\mathrm{L} 2$ representations.

Finally, our results suggest that different time-locks should routinely be applied in the analysis of EEG signals in psycholinguistic experiments.

\section{Acknowledgements}

The data used in this work was collected in a project funded by an ERC consolidator grant (284108) to Mirjam Ernestus. 


\section{References}

[1] A. Newell and H. Simon, Human problem solving. Englewood Cliffs, NJ: Prentice Hall, 1972.

[2] A. Rangel, C. Camerer, and P. Montague, "A framework for studying the neurobiology of value-based decision making," Nature Re views Neuroscience, pp. 545 - 556, 2008.

[3] V. Marian and M. Spivey, "Competing activation in bilingual language processing: Within- and between-language competition," Bilingualism: Language and Cognition, vol. 6, no. 2, p. 97-115, 2003.

[4] M. Finkbeiner, M. Coltheart, and V. Coltheart, "Pointing the way to new constraints on the dynamical claims of computational models," Journal of Experimental Psychology: Human Perception and Performance, vol. 40, no. 1, pp. $172-$ 185, 2014. [Online]. Available: https://doi.org/10.1037/a0033169

[5] G. Suri, J. J. Gross, and J. L. McClelland, "Value-based decision making: An interactive activation perspective," Psychological Review, vol. 127, no. 2, pp. 153 - 185, 2020.

[6] A. Strauß, S. Kotz, M. Scharinger, and J. Obleser, "Alpha and theta brain oscillations index dissociable processes in spoken word recognition," Neuroimage, vol. 97, pp. 387 - 395, 2014.

[7] J. Brennan, C. Lignos, D. Embick, and T. Roberts, "Spectrotemporal correlates of lexical access during auditory lexical decision," Brain and Language, vol. 133, pp. 39-46, 2014.

[8] L. Drijvers, K. Mulder, and M. Ernestus, "Alpha and gamma band oscillations index differential processing of acoustically reduced and full forms," Brain and Language, vol. 153-154, pp. 27-37, 2016.

[9] T. B. O'Rourke and P. J. Holcomb, "Electrophysiological evidence for the efficiency of spoken word processing," Biological Psychology, vol. 60, no. 2, pp. 121 - 150, 2002.

10] L. ten Bosch, L. Boves, and M. Ernestus, "DIANA: towards computational modeling reaction times in lexical decision in north american english," in Proc. Interspeech 2015, Dresden, Germany, 2015, pp. $1576-1580$.

111] R. G. O'Connell, P. M. Dockree, and S. P. Kelly, "A supramoda accumulation-to-bound signal that determines perceptual decisions in humans," Nature Neuroscience, vol. 15, no. 12, pp. 1730 $-1735,2012$

[12] M. van Vugt, M. Beulen, and N. Taatgen, "Relation between centro-parietal positivity and diffusion model parameters in both perceptual and memory-based decision making," Brain Research, no. 1715 , pp. 1 - 12, 2019.

[13] K. Mulder, G. Brekelmans, and M. Ernestus, "The processing of schwa reduced cognates and noncognates in non-native listeners of English," in Proceedings of the 18th International Congress of Phonetic Sciences [ICPhS 2015], 2015.

[14] A. Strauß and J.-L. Schwartz, "The syllable in the light of motor skills and neural oscillations," Language, Cognition and Neuroscience, vol. 32, no. 5, pp. 562-569, 2017.

[15] D. Norris and J. McQueen, "Shortlist B: A Bayesian model of continuous speech recognition," Psychological Review, vol. 115, pp. $357-395,2008$.

[16] D. Arnold, F. Tomaschek, K. Sering, F. Lopez, and R. Baayen, "Words from spontaneous conversational speech can be recognized with human-like accuracy by an error-driven learning algorithm that discriminates between meanings straight from smart acoustic features, bypassing the phoneme as recognition unit," PLoS ONE, vol. 12, no. 4:e0174623, 2017. [Online]. Available: https://doi.org/10.1371/journal.pone.0174623

[17] A. Kösem and V. van Wassenhove, "Distinct contributions of lowand high-frequency neural oscillations to speech comprehension," Language, Cognition and Neuroscience, vol. 32, no. 5, pp. 536544, 2017.

[18] W. Van Heuven, P. Mandera, E. Keuleers, and M. Brysbaert, "Subtlex-UK: A new and improved word frequency database for British English," Quarterly Journal of Experimental Psychology, vol. 67, pp. 1176-1190, 2014
[19] Brain Products, BrainVision Analyzer User Manual, Version 1.03, Brain Vision LLC, Morrisville, NC, 2006, page $125 \mathrm{ff}$.

[20] M. Crosse, G. Di Liberto, A. Bednar, and E. Lalor, "The multivariate temporal response function (mTRF) toolbox: A MATLAB toolbox for relating neural signals to continuous stimuli," Frontiers in Human Neuroscience, vol. 10, 2016, article 604.

[21] K. Mulder, L. ten Bosch, and L. Boves, "Analyzing EEG signals in auditory speech comprehension using temporal response functions and generalized additive models," in Proc. Interspeech 2018, 2018, pp. 1452-1456. [Online]. Available: http://dx.doi.org/10.21437/Interspeech.2018-1676

[22] H. Luo and D. Poeppel, "Phase patterns of neuronal responses reliably discriminate speech in human auditory cortex," Neuron, vol. 54, pp. $1001-1010,2007$

[23] L. ten Bosch, K. Mulder, and L. Boves, "Phase synchronization between eeg signals as a function of differences between stimuli characteristics," in Proc. Interspeech 2019, Graz, Austria, 2019. pp. $1213-1217$.

[24] A. Nijveld, L. ten Bosch, and M. Ernestus, "ERP signal analysis with temporal resolution using a time window bank," in Proc. Interspeech 2019, Graz, 2019, pp. 1208 - 1212.

[25] K. Winsler, K. J. Midgley, J. Grainger, and P. J. Holcomb, "An electrophysiological megastudy of spoken word recognition," Language, Cognition and Neuroscience, pp. 1 - 22, 2018.

[26] D. Bates, M. Mächler, B. Bolker, and S. Walker, "Fitting linear mixed-effects models using lme4," Journal of Statistical Software, vol. 67, no. 1, pp. 1-48, 2015.

[27] R Core Team, R: A Language and Environment for Statistical Computing, R Foundation for Statistical Computing, Vienna, Austria, 2013. [Online]. Available: http://www.R-project.org

[28] L. ten Bosch, M. Ernestus, and L. Boves, "Analyzing reaction time sequences from human participants in auditory experiments," in Proc. Interspeech 2018, 2018, pp. 971-975. [Online]. Available: http://dx.doi.org/10.21437/Interspeech.2018-1728

[29] R. Ratcliff, P. L. Smith, S. D. Brown, and G. McKoon, "Diffusion decision model: Current issues and history," Trends Cogn Sci., vol. 20, no. 4, pp. 260-281, 2016.

[30] A. Bürki and M. Gaskell, "Lexical representation of schwa words Two mackerels, but only one salami," Journal of Experimental Psychology: Learning Memory and Cognition, vol. 38, no. 3, pp. $617-631,2012$.

[31] P. LoCasto and C. Connine, "Rule-governed missing information in spoken word recognition: Schwa vowel deletion," Perception \& Psychophysics, vol. 64, pp. $208-219,2002$.

[32] M. K. van Vugt, P. Simen, and J. D. Cohen, "Trial-by-trial adaptation of decision making performance - a model-based EEG analysis," in Interdisciplinary Perspectives on Cognition, Education, and the Brain, J. Ozyurt, A. Anschütz, S. Bernholt, and J. Lenk, Eds. Oldenburg: Hanse Studies, 2011, vol. 7, pp. $139-148$ 